\title{
Emotional Stroop task: effect of word arousal and subject anxiety on emotional interference
}

\author{
Thomas Dresler $\cdot$ Katja Mériau $\cdot$ \\ Hauke R. Heekeren $\cdot$ Elke van der Meer
}

Received: 1 October 2007 / Accepted: 25 June 2008 / Published online: 18 July 2008

(C) The Author(s) 2008

\begin{abstract}
Inconsistent findings regarding the emotional Stroop effect in healthy subjects may be explained by confounding effects of stimulus valence and arousal, as well as individual differences in anxiety. We examined reaction time data in a healthy sample using the emotional Stroop task while carefully matching arousal level of positive and negative words. Independent of valence, emotional relative to neutral words elicited emotional interference, indicating that arousal determines emotional interference. Independent of valence, emotional words were better re-called and recognized than neutral words. Individual differences in state anxiety were associated with emotional interference, that is, emotional interference was enhanced in subjects with high state anxiety. There was no influence of trait anxiety. These findings indicate that word arousal produces emotional interference independent of valence. State anxiety
\end{abstract}

Thomas Dresler and Katja Mériau have contributed equally.

T. Dresler $(\bowtie) \cdot$ K. Mériau $\cdot$ H. R. Heekeren

Department of Neurology, Neuroscience Research Center, Berlin NeuroImaging Center, Charité University Medicine Berlin, Campus Mitte, Charitéplatz 1, 10117 Berlin, Germany

e-mail: thomas.dresler@charite.de

T. Dresler · K. Mériau · E. van der Meer

Department of Psychology,

Humboldt University, Berlin, Germany

K. Mériau · H. R. Heekeren

Max Planck Institute for Human Development, Berlin, Germany

Present Address:

T. Dresler

Psychophysiology and Functional Imaging,

Department of Psychiatry, Psychosomatics and Psychotherapy,

University Würzburg, Füchsleinstr. 15,

97080 Würzburg, Germany exacerbates interference of emotional words by further biasing attention towards emotionally salient stimuli.

\section{Introduction}

Emotional processes have an essential influence on cognitive processes. One approach to investigate interference effects of emotional material on cognitive processing is to use the emotional Stroop task (e.g. Williams et al. 1996) in which subjects have to name the ink colour of word stimuli as fast and accurately as possible, while at the same time ignoring the word meaning. Slowing of naming the ink colour of emotional as compared to neutral words represents the emotional interference effect and indicates biasing of attentional resources towards the emotionally salient information (Williams et al. 1997). Such an attentional bias for emotional stimuli was also shown with pictures (Schimmack 2005) and other tasks used in attention research, e.g. the dot probe task (MacLeod et al. 1986) and the spatial cueing task (see Bar-Haim et al. 2007). So far, however, the emotional Stroop task is the most frequently used experimental design to investigate attentional bias (Williams et al. 1996).

To date, it remains unclear whether valence or arousal of emotional material determines interference (Schimmack 2005). Pratto and John (1991) repeatedly found that colournaming latencies were longer for negative as compared to positive words (categorical negativity theory). Those authors take interference as an indicator of automatic processing of negative stimuli as they have greater significance for the individual (Pratto and John 1991). This attentional negativity bias has been supported by a number of studies using the emotional Stroop task (e.g. McKenna and Sharma 1995; Sharma and McKenna 2001). There is less consistent evidence for an interference effect of positive words, though (Dalgleish 1995; 
Koven et al. 2003; Martin et al. 1991; Pratto and John 1991; Ruiz-Caballero and Bernandez 1997). A study by Pratto (1994) revealed that negative and positive words that were both high in arousal elicited emotional interference. Similarly, Schimmack (2005) reported that interference effects elicited by emotional pictures depended solely on their arousal level and not on their valence, and Lang et al. reported that subjects choose to look longer at arousing pictures independently of their valence (Lang et al. 1993). Furthermore, in a recent study using a spatial attention task with emotional pictures, high levels of stimulus arousal as compared to low levels of stimulus arousal were associated with an attention shift within the visual space; an effect that was found irrespective of stimulus valence (Robinson and Compton 2006). Finally, the affective modulation of the attentional blink was related to the arousal level of word stimuli rather than to their valence (Anderson 2005).

In conclusion, there is ample evidence to suggest that the arousal level, and not valence of emotional stimuli matters in producing emotional interference. Indeed, a direct comparison of emotional words with high vs. low arousal showed that in healthy individuals highly arousing stimuli elicited greater interference than stimuli with low arousal, however, the effect was more pronounced for negative than for positive words (Compton et al. 2003). Consequently, this requests a thorough control of arousal levels of emotional stimuli of different valences, especially because negative emotional stimuli are frequently more arousing than positive emotional stimuli (Lang et al. 1993). For instance, the covariation between arousal and valence appears to be stronger for unpleasant adjectives than for pleasant adjectives and regression lines relating arousal to unpleasant or pleasant valence differ in steepness and slope (Herbert 2007; Ito et al. 1998). Thus, it is more likely for negative words to be associated with high arousal than for positive words (Lang et al. 1997). In that sense, a meta-analysis concerning the impact of positive stimuli in emotional Stroop and dot probe tasks (Ruiz-Caballero and Bernandez 1997) only found partial evidence for an attentional bias for positive words. However, the included studies were quite diverse (non-clinical anxious subjects, clinical subjects, anxiety induction vs. low/high trait anxious subjects) and the actual arousal level of words was not controlled systematically.

To investigate whether the emotional interference in healthy subjects is mediated by valence or arousal, we used negative and positive words that were comparable in arousal level in an emotional Stroop task.

Individual differences in affectivity, such as anxiety in healthy subjects were also shown to influence emotional interference and may account for inconsistent findings concerning the emotional interference effect (Bar-Haim et al. 2007; Egloff and Hock 2001; Richards et al. 1992). To date, it remains unclear whether enhanced emotional interference in healthy anxious subjects is rather a function of stable personality traits (e.g. trait anxiety) or of transient mood states (e.g. state anxiety). Trait anxiety reflects a more general and relatively stable tendency to respond with anxiety, while state anxiety represents a more transitory and temporary condition of anxiety that can differ between situations (Laux et al. 1981). Whereas the influence of trait anxiety has been investigated (Dalgleish 1995; Richards et al. 1992; see Williams et al. 1996 for a review), only few studies examined the influence of state anxiety or interactive effects of trait and state anxiety on emotional interference and produced inconsistent results (Broadbent and Broadbent 1988; Egloff and Hock 2001; see Bar-Haim et al. 2007). This may be due to methodological differences in the investigation of effects of state anxiety as natural mood studies typically yielded stronger effects than mood induction studies (Rusting 1998). To address this issue we assessed individual differences in state and trait anxiety in a healthy sample and their influence on emotional interference.

Emotional material also has an impact on memory processes: emotional stimuli are better re-called and recognized than neutral stimuli, an effect that has been labelled memory enhancement effect. This has been found equally for pictures, words, sentences, autobiographical events, and stories (Hamann 2001; Kensinger and Corkin 2003; Linton 1982; Waagenar 1986; Rubin 2005). There is evidence that arousal is a driving force behind these memory effects, as pictures that are high in arousal are better remembered than pictures that are low in arousal (Bradley et al. 1992). To further assess the memory enhancing effects of the emotional stimuli in the present study we applied a surprise re-call and recognition task following the emotional Stroop task.

The present study investigated whether emotional interference is produced by word valence or word arousal. We also explored the influence of trait and state anxiety and their interaction on emotional interference in a healthy sample. We hypothesize that the emotional interference effect is mediated by arousal and not valence, that is, colour-naming latencies for negative and positive words will not differ when controlling for word arousal. We predict an influence of trait and state anxiety on emotional interference.

\section{Methods}

\section{Subjects}

Fifty university students (30 female; $25.2 \pm 3.3$ years, mean age \pm standard deviation, SD) participated in the study. Subjects were undergraduate or graduate students. Individual differences in state and trait anxiety were assessed using the State-Trait Anxiety Inventory (STAI, 
Laux et al. 1981). To avoid that potentially stress-inducing effects of the experimental situation would distort results of the questionnaires, these were applied right before the actual experiment.

\section{Stimulus material}

In a pilot study ( $n=47$; age $27.0 \pm 7.6$ years) 300 selected nouns were rated with regard to valence and arousal using seven-point rating scales. Twenty negative words (e.g. burglary, victim; valence $-2.21 \pm 0.38$; arousal $4.26 \pm 0.12$ ), 20 positive words (e.g. humour, party; valence $1.81 \pm 0.58$; arousal $4.27 \pm 0.34$ ) and 20 neutral words (e.g. oats, coil; valence $0.12 \pm 0.25$; arousal $1.66 \pm 0.35$ ) were chosen for the emotional Stroop task. Positive and negative words did not differ with regard to arousal [Bonferroni-corrected $t$-test $t(38)=0.11, P=1.0]$; however, they were more arousing than neutral words [negative vs. neutral: $t(38)=31.07$, $P<0.01$; positive vs. neutral: $t(38)=23.80, P<0.01]$. Negative, positive, and neutral words were comparable for number of letters $[F(2,57)=1.14, \mathrm{MSE}=2.65, P=0.33]$, syllables $\quad[F(2,57)=0.06, \quad \mathrm{MSE}=0.26, \quad P=0.94] \quad$ and frequency [The CELEX database, $F(2,57)=0.06$, MSE $=$ 2313.39, $P=0.94]$, respectively.

\section{Procedure}

Emotional and neutral words in red, green, yellow, and blue colour were displayed separately on a black computer screen. The luminance of colours of words was comparable (3 Candela $/ \mathrm{m}^{2}$ ) as measured with a luminance meter (Gossen Company, type MAVOLUX digital). Subjects had to indicate the ink colour of the presented words using four keys of a standard computer keyboard, while ignoring the meaning of the word. Words were presented on the screen until a response was given. Assignment of colours to keys was counterbalanced across subjects.

The experiment consisted of two runs and each run contained four blocks of neutral, positive, and negative words, respectively (in total twelve blocks). A block design was chosen because it proved to be the best method to elicit emotional interference in healthy subjects in comparison to a random and event-related stimulus presentation (Bar-Haim et al. 2007). Order of blocks was pseudorandomized in an $\mathrm{ABCBAC}$ fashion $(\mathrm{A}=$ neutral, $\mathrm{B}=$ positive, $\mathrm{C}=$ negative) to avoid consecutive presentation of blocks of the same valence. Within each block ten words (trials) were presented. Order of trials was pseudo-randomized to avoid that consecutive trials elicited the same manual response. In total, subjects were exposed to 80 negative, 80 positive, and 80 neutral words. Before each block a fixation cross was presented for $6 \mathrm{~s}$; trials were separated by a fixation cross displayed for $1.5 \mathrm{~s}$.
As training, subjects performed the classical Stroop task prior to the experiment. The classical Stroop task consisted of two blocks: during the first block 60 congruent items (12 practice trials, 48 test trials, e.g. the word "red" written in red colour) and during the second block 48 incongruent items (e.g. the word "red" written in blue colour) were displayed.

Subsequent to the emotional Stroop task, subjects were asked to re-call and recognize the presented words in a surprise re-call and recognition task. In the surprise re-call task they were asked to write down all the words they remembered. In the surprise recognition task, each of the 60 word stimuli from the experiment was presented with a distractor item and subjects had to indicate which one was originally presented in the experiment (e.g. oats vs. boat). Presentation of word pairs was pseudo-randomized, as was the screen position (top or bottom) of the target word. Distractor items were matched with target items in terms of valence and grammatical category (noun). Target and distractor items did not differ for number of letters $[t(118)=0.28, P=0.78]$, syllables $[t(118)=0.17, P=0.86]$, and frequency [The CELEX database, $t(112)=1.55$, $P=0.12]$.

\section{Results}

Reaction time data and error rates

Colour-naming errors (1.8\%) and consecutive trials were eliminated from the data set. Individual reaction times of more than two SDs from a subject's mean were also excluded from further analysis $(4.9 \%)$. Mean reaction times and error rates for negative, positive, and neutral words, respectively, are displayed in Table 1.

Reaction time data and error rate data were submitted to a repeated measures ANOVA. An alpha level of 0.05 was set for all statistical tests. Reaction times were influenced by the experimental conditions $[F(2,98)=7.07, \mathrm{MSE}=591.41$,

Table 1 Reaction times (in milliseconds), error rates, re-call and recognition rates for negative, positive, and neutral words (mean \pm standard deviation; $n=50$ )

\begin{tabular}{llll}
\hline & Negative & Positive & Neutral \\
\hline Reaction times & 733.59 & 736.41 & 719.35 \\
& $( \pm 132.70)$ & $( \pm 126.07)$ & $( \pm 109.37)$ \\
Error rates & $1.68( \pm 1.91)$ & $1.26( \pm 1.32)$ & $1.48( \pm 0.85)$ \\
Re-call rates & $3.44( \pm 1.85)$ & $3.88( \pm 2.23)$ & $1.70( \pm 1.74)$ \\
$\begin{array}{l}\text { Recognition } \\
\text { rates }\end{array}$ & $17.50( \pm 1.94)$ & $17.06( \pm 2.07)$ & $15.70( \pm 2.72)$ \\
\hline
\end{tabular}


$P<0.01$ ], that is, emotional words elicited longer reaction times than neutral words $[t(49)=3.11, P<0.01]$. With regard to valence, Bonferroni-corrected post hoc comparisons revealed that reaction times for negative and positive words were significantly longer than for neutral words [negative $t(49)=2.49, P<0.05 ;$ positive $t(49)=3.43$, $P<0.01]$. Reaction times did not differ significantly between negative and positive words $[t(49)=0.77, P=1.0]$. There was no effect of experimental conditions on error rates $[F(2,98)=1.31, \mathrm{MSE}=1.69, P=0.28]$, that is, there was no speed-accuracy trade-off.

To investigate the magnitude of the emotional Stroop effect, we calculated an emotional interference score, defined as the difference between mean reaction times for neutral and negative words $\left(\mathrm{RT}_{\text {negative }}-\mathrm{RT}_{\text {neutral }}=14.25 \mathrm{~ms}\right.$, $\mathrm{SD}=40.49$ ) and for neutral and positive words $\left(\mathrm{RT}_{\text {positive }}-\mathrm{RT}_{\text {neutral }}=17.06 \mathrm{~ms}, \mathrm{SD}=35.16\right)$, respectively (see Table 1). The mean interference score for emotional words independent of valence was $\mathrm{RT}_{\text {emotional }}$ $\mathrm{RT}_{\text {neutral }}=15.65 \mathrm{~ms}(\mathrm{SD}=35.63)$. We used Cohen's formula for dependent measures $\left(d=\mathrm{M}_{1}-\mathrm{M}_{2} / \mathrm{SD}_{\text {Difference }} \times\right.$ $\sqrt{ } 2)$ to calculate the effect sizes of interference scores (Cohen 1988). The effect sizes were $d=0.49$ for negative vs. neutral, $d=0.68$ for positive vs. neutral and $d=0.61$ for emotional vs. neutral words and are comparable to those reported by Bar-Haim et al. (2007).

Reliability of emotional interference scores was tested using the split-half method. Interference scores of emotional stimuli from the first half of each run were correlated with the interference scores from the second half. Split-half reliability amounted to 0.76 (Spearman-Brown corrected), which is above the reliability scores for emotional interference scores using a retest design with a 1-week interval (Eide et al. 2002). Split-half reliability was 0.75 for negative and 0.65 for positive interference.

To summarize, reaction times did not differ between negative and positive words that were equal in arousal. Reaction times for negative and positive words, however, were both longer than for neutral words.

Individual differences in trait and state anxiety

Individual differences in trait and state anxiety were assessed using the STAI (Laux et al. 1981). The mean trait anxiety score in our sample was $36.42(\mathrm{SD}=5.83)$, the mean state anxiety score $33.56(\mathrm{SD}=6.53)$. To investigate effects of both trait and state anxiety on emotional interference we conducted a regression analysis with state and trait anxiety and its interaction term (after z-standardization) as continuous predictors of emotional interference. Compared to bivariate correlational analyses, this approach has the advantage of estimating the particular influence of each predictor while controlling for the influence of the other predictors at the same time. As we hypothesized a positive association of anxiety scores with interference, we applied one-tailed significance testing to the data. The regression model was significant $\left[F(3,46)=3.64, P=0.02, R^{2}=0.19\right]$ and revealed a significant effect of state anxiety $(\beta=0.41$, $P=0.01)$, but no effect of trait anxiety or the interaction term (both $\beta<0.05$, ns). For the valence-dependent interference separate analyses were conducted with positive and negative interference as the to-be-predicted variables. The regression model for positive interference proved to be significant $\left[F(3,46)=5.12, P<0.01, R^{2}=0.25\right]$ with state anxiety being the only significant predictor $(\beta=0.44$, $P=0.01$ ). The model for negative interference failed to reach significance $\left[F(3,46)=1.99, P=0.13, R^{2}=0.12\right]$, however, the regression coefficient of state anxiety remained significant $(\beta=0.33, P<0.05)$. The failure to demonstrate an effect of trait anxiety may be explained by an only minor variation of trait anxiety scores in our sample (SD in $t$-norm equivalent 5.98) compared to the norm sample (Laux et al. 1981).

To summarize, regression analyses results indicate that individual differences in state anxiety influenced emotional interference, trait anxiety, however, did not and there were no interactive effects of state and trait anxiety.

Re-call and recognition data

There was a significant effect of experimental conditions on free re-call $[F(2,98)=31.04, \operatorname{MSE}=2.14, P<0.01]$. Bonferroni-corrected post hoc $t$-tests revealed that negative and positive words were better re-called than neutral words [negative vs. neutral $t(49)=6.13, P<0.01$; positive vs. neutral: $t(49)=6.89, P<0.01$ ] (see Table 1$)$. There was no difference between negative and positive words $[t(49)=$ $1.60, P=0.35]$.

Recognition of negative, positive, and neutral words was above chance $[\operatorname{ts}(49)>24.00, P \mathrm{~s}<0.01]$. There also was a significant effect of experimental conditions on recognition $[F(2,98)=16.22, \operatorname{MSE}=2.71, P<0.01]$. Post hoc $t$-tests revealed that negative and positive words were better recognized than neutral words [negative vs. neutral $t(49)=$ 4.95, $P<0.01$; positive vs. neutral $t(49)=4.13, P<0.01$ ] (see Table 1). There was no difference between negative and positive words $[t(49)=1.51, P=0.41]$.

To summarize, re-call and recognition scores did not differ between negative and positive words that were equal in arousal. However, negative and positive words were better re-called and recognized than neutral words.

To control for the possibility of attention-mediated (instead of arousal-mediated) memory effects we conducted regression analyses with re-call and recognition scores for each word (sum of free re-call and recognition scores) as the to-be-predicted variables, and with the mean reaction 
times and arousal scores per word calculated across all participants as predictors (Lang et al. 1993). The regression model was significant for both re-call $[F(2,57)=18.09$, $\left.P<0.01, R^{2}=0.38\right]$ and recognition scores $[F(2,57)=5.98$, $\left.P<0.01, R^{2}=0.17\right]$. For re-call scores both arousal $(\beta=0.22, P<0.05)$ and reaction time $(\beta=0.52, P<0.01)$ were significant predictors. For the recognition score model, however, only arousal scores proved to be a significant predictor $(\beta=0.42, P<0.01)$, indicating that attention-mediating effects were irrelevant with regard to recognition performance.

\section{Discussion}

In the present study, we investigated whether emotional interference is produced by word valence or word arousal. Consistent with our hypothesis we found an emotional interference effect for emotional words, independent of word valence. The surprise re-call and recognition task revealed that emotional words were better re-called and recognized than neutral words and that the recognition effect was not mediated by attention, but by arousal. We also investigated the influence of individual differences in state and trait anxiety on emotional interference. State anxiety was associated with emotional interference, that is, emotional interference was enhanced in subjects with high state anxiety. Trait anxiety had no influence on emotional interference.

Arousing word stimuli, independently of their valence produced emotional interference, that is, ink colour-naming latencies for both positive and negative compared to neutral words were increased.

The effect sizes for the interference effects of emotional words (about 0.50 ) in the present study are consistent with the effect sizes reported in a meta-analysis on emotional interference (Bar-Haim et al. 2007). Bar-Haim et al. (2007) report a reliable attentional bias for different paradigms in anxious subjects. Interestingly, with regard to the emotional Stroop task they report that only blocked presentation of emotional words elicits emotional interference in non-clinical control subjects whereas mixed presentation did not. Similarly, in our study emotional interference occurred with a blocked presentation of emotional words of the same valence.

The present findings do not support the categorical negativity theory, which postulates that only stimuli automatically evaluated as negative attract attention (Pratto and John 1991). The results of the current study add to the increasing evidence that arousal, and not valence, produces emotional interference in information processing tasks such as the emotional Stroop task (Compton et al. 2003; Schimmack 2005), the attentional blink paradigm (Anderson 2005), or spatial attention paradigms (Robinson and Compton
2006). In contrast to the categorical negativity theory, the arousal theory postulates that the first appraisal of a stimulus is related to arousal and not valence (Anderson 2005; Lang et al. 1993, Schimmack 2005). The present findings, however, go beyond the results by Compton et al. (2003) by revealing an interference effect not only for high vs. low arousing negative words, but also for highly arousing words independently of their valence, that is, for both positive and negative words. Schimmack (2005) showed that arousal levels of very unpleasant pictures and pleasant pictures of opposite-sex models predicted interference effects. This arousal effect is in line with the finding of Robinson and Compton (2006). The researchers independently varied valence and arousal of pictorial stimuli and found that highly arousing pictures, independent of their valence, resulted in a leftward visual shift of attention indicated by faster responses to subsequently presented targets in the left compared to the right visual field. The present findings accord well with these results, extending the assumptions of the arousal theory from visual to verbal material and furthermore show that interference is also produced by positive stimuli other than sexually arousing stimuli that may represent a very distinctive incentive.

The interference effect is thought to reflect increased allocation of attentional resources to emotional stimuli, especially negative ones (Pratto and John 1991). The present findings, however, indicate that allocation of attentional resources is rather mediated by arousal of stimuli. This may facilitate further and more in-depth processing of relevant stimuli to guide adaptive behaviour of an individual (Schimmack 2005; Pratto and John 1991). Processing stimuli in greater depth or on a more elaborated level may increase the accessibility of the stored representations and result in increased re-call and recognition.

This "emotional memory enhancement effect" was also shown in the present study. During the free re-call and recognition tasks, significantly more emotional words than neutral words were re-called and recognized. The fact, that as many positive as negative words were re-called and recognized again indicates that the arousal dimension has a greater or at least more immediate impact than the valence dimension and is consistent with the reaction time data.

The present results are in line with findings indicating that memory performance is most sensitive to the arousal level of the stimuli, that is, pictures that were rated as highly arousing are better remembered than less arousing pictures (e.g. Bradley et al. 1992).

The better memory performance found for highly arousing words (independent of valence) might be mediated by attention. That is, the longer exposure times of emotional as compared to neutral words as indicated by prolonged reaction times during colour-naming of emotional words may allow for longer consolidation processes which, in turn, 
promote better re-call and recognition of these words. However, this was only partly true for the re-call performance, as longer reaction times and higher arousal scores predicted re-call scores for emotional words. This can be interpreted as a better re-call performance for words that have been processed to greater depth due to longer exposure time. Conversely, the regression analysis for the recognition scores revealed that only the arousal scores but not word reaction times (as a measure of attention) were significant predictors of the recognition scores, indicating that recognition performance is affected by word arousal and not mediated by attention. However, there is evidence that disruptive effects of emotional Stroop words (e.g. memory effects) are mediated by mechanisms that last longer than the response interval of the actual trial (e.g. McKenna and Sharma 2004). We can therefore not rule out that a better recognition performance for emotional words was due to a "longer consolidation process" in the inter-trial interval.

What do these results tell us, that the effect of arousal on recognition was not mediated by attention? There is behavioural evidence for automatic emotional perception in the absence of conscious awareness. For instance, several studies have demonstrated that subjects can make good/bad evaluative judgements of subliminally presented words at better-than-chance levels (for a review see Pratto 1994). Such automatic emotional word perception is likely to be mediated by a subcortical circuit involving the amygdala that acts as a detector of emotional word content at a very early stage of processing (Landis 2006; Naccache et al. 2005). More specifically, the amygdala is critical for intact recognition of emotional arousal from words, sentences, and facial expressions, but not for intact recognition of emotional valence (Adolphs et al. 1999). Indeed, distinct neural networks contribute to emotional memory enhancement for arousing words vs. valenced, non-arousing words: memory enhancement for arousing words is mediated by an amygdalar-hippocampal-network, whereas memory enhancement effect for valenced, non-arousing words is supported by a prefrontal-hippocampal network associated with controlled, self-generated encoding processes (Kensinger and Corkin 2004).

In conclusion, the dimensions of valence and arousal both influence memory-encoding processes, however, their effects may be differentially mediated (Kensinger and Corkin 2004). That is, effects of arousal are mediated by a subcortical network involving the amygdala that automatically activates in response to subliminally presented emotional word stimuli (Adolphs et al. 1999; Naccache et al. 2005). These findings speak for a memory enhancement effect for emotional words that is primarily mediated by the arousal dimension occurring before attentional processes step in. Taken together, the present experiment revealed that arousal and not valence influenced colour- naming latencies as well as re-call and recognition performance.

Individual differences in state anxiety were associated with the emotional interference effect, that is, subjects with higher state anxiety showed increased emotional interference. This finding is in accordance with other studies investigating the effect of state anxiety on emotional interference (see Bar-Haim et al. 2007) and supports the notion that anxiety is associated with automatic allocation of attentional resources to threat-related stimuli (Williams et al. 1997) and emotional stimuli in general, respectively, thereby binding cognitive resources. State anxiety represents a temporary emotional state associated with psychophysiological responses to allow for immediate behavioural reactions, that is, state anxiety appears to be more preparatory for action than trait anxiety. An acute emotional state such as state anxiety may denote a greater behavioural urgency that has greater impact on cognitive performance. Additional assessment of psychophysiological arousal may clarify whether bodily arousal plays an essential role in influencing cognitive performance or, if absent, whether cognitive arousal or state of alertness without concomitant psychophysiological arousal may have similar effects.

In contrast to state anxiety, individual differences in trait anxiety had no influence on neither negative nor positive emotional interference in the present study. Similarly, Martin et al. (1991) and Egloff and Hock (2001) also failed to find an effect of trait anxiety on emotional interference in a healthy sample. How can this finding be explained? Subjects with high trait anxiety may have developed emotion regulative strategies or coping mechanisms to regulate their mood. This may be especially the case in situations where individuals are motivated to perform well on a difficult task as in a laboratory experiment. Moreover, trait anxiety represents a stable tendency to respond with anxiety, but does not imply that the individual is in a constant state of worry and apprehension that influences cognitive performance. The failure to demonstrate an association between trait anxiety and emotional interference may also relate to the little range in trait anxiety scores in our healthy sample as a low variation impedes the demonstration of an association of trait anxiety with interference magnitude.

Rusting postulated three conceptual frameworks of how personality trait and mood states may influence emotional processing (Rusting 1998). First, personality traits and mood states have independent effects; second, personality traits moderate the influence of mood states on emotional processing (i.e. mood states may have different outcomes depending on the personality traits); third, influences of personality traits on emotional processing are mediated by current mood states (i.e. traits render one more susceptible to certain mood states which then influence emotional processing) (Rusting 1998). As the present study simultaneously 
investigated state and trait anxiety and their interaction effects we can infer from the present data that state and trait anxiety have independent effects on emotional processing.

In conclusion, the present data indicate an exclusive effect of state anxiety on emotional processing. Cognitive theories explaining the attentional bias in anxiety differ in whether they assume an effect of anxiety on earlier automatic stages of processing (i.e. hypervigilance) or on later stages of processing (i.e. avoidance of emotional information or impaired ability to disengage from threatening information). Whether state anxious subjects show a hypervigilance towards emotional information cannot be inferred from the present data. The data clearly indicate, however, that state anxiety is associated with a difficulty in disengaging attention from emotional material.

Further research on the interference effect of emotional material should consider subject characteristics (e.g. affectivity) and stimulus characteristics (e.g. arousal). Assessment of psychophysiological changes may further clarify the significance of dimensions of arousal such as cognitive arousal vs. bodily arousal in producing interference, especially in cases where self-reported and objectively measured arousal dissociate as they do in repression.

Acknowledgments This study was financially supported by a grant from the BMBF (Berlin NeuroImaging Center, BNIC). We gratefully acknowledge the help of Philipp Kazzer with programming the experiment.

Open Access This article is distributed under the terms of the Creative Commons Attribution Noncommercial License which permits any noncommercial use, distribution, and reproduction in any medium, provided the original author(s) and source are credited.

\section{References}

Adolphs, R., Russell, J. A., \& Tranel, D. (1999). A role for the human amygdala in recognizing emotional arousal from unpleasant stimuli. Psychological Science, 10, 167-171.

Anderson, A. K. (2005). Affective influences on the attentional dynamics supporting awareness. Journal of Experimental Psychology: General, 134, 258-281.

Bar-Haim, Y., Lamy, D., Pergamin, L., Bakermans-Kranenburg, M. J., \& van IJzendoorn, M. H. (2007). Threat-related attentional bias in anxious and nonanxious individuals: A meta-analytic study. Psychological Bulletin, 133, 1-24.

Bradley, M. M., Greenwald, M. K., Petry, M. C., \& Lang, P. J. (1992). Remembering pictures: Pleasure and arousal in memory. Journal of Experimental Psychology: Learning, Memory, \& Cognition, 18, 379-390.

Broadbent, D. E., \& Broadbent, M. (1988). Anxiety and the attentional bias: State and trait. Cognition \& Emotion, 2, 165-183.

Cohen, J. (1988). Statistical power analysis for the behavioral sciences (2nd ed.). Hillsdale, NJ: Erlbaum.

Compton, R. J., Banich, M. T., Mohanty, A., Milham, M. P., Herrington, J., Miller, G. A., et al. (2003). Paying attention to emotion: An fMRI investigation of cognitive and emotional stroop tasks. Cognitive. Affective \& Behavioral Neuroscience, 3, 81-96.
Dalgleish, T. (1995). Performance on the emotional Stroop task in groups of anxious, expert, and control subjects: A comparison of computer and card presentation formats. Cognition \& Emotion, 9 , 341-362.

Egloff, B., \& Hock, M. (2001). Interactive effects of state anxiety and trait anxiety on emotional Stroop interference. Personality and Individual Differences, 31, 875-882.

Eide, P., Kemp, A., Silberstein, R. B., Nathan, P. J., \& Stough, C. (2002). Test-retest reliability of the emotional Stroop task: Examining the paradox of measurement change. The Journal of Psychology, 136, 514-520.

Hamann, S. (2001). Cognitive and neural mechanisms of emotional memory. Trends in Cognitive Sciences, 5, 394-400.

Herbert, C. (2007). Emotional words obtain priority in processing: Evidence from event-related brain potential and functional magnetic resonance imaging studies. Dissertation, University of Konstanz, Konstanz.

Ito, T. A., Cacioppo, J. T., \& Lang, P. J. (1998). Eliciting affect using the International Affective Picture System: Bivariate evaluation and ambivalence. Personality and Social Psychology Bulletin, 24, 855-879.

Kensinger, E. A., \& Corkin, S. (2003). Memory enhancement for emotional words: Are emotional words more vividly remembered than neutral words? Memory \& Cognition, 31, 1169-1180.

Kensinger, E. A., \& Corkin, S. (2004). Two routes to emotional memory: Distinct neural processes for valence and arousal. Proceedings of the National Academy of Sciences of the USA, 101, 33103315 .

Koven, N. S., Heller, W., Banich, M. T., \& Miller, G. A. (2003). Relationships of distinct affective dimensions to performance on an emotional stroop task. Cognitive Therapy and Research, 27, 671680 .

Landis, T. (2006). Emotional words: what's so different from just words? Cortex, 42, 823-830.

Lang, P. J., Bradley, M. M., \& Cuthbert, B. N. (1997). Motivated attention: Affect, activation and action. In P. J. Lang, R. F. Simons \& M. T. Balaban (Eds.), Attention and orienting: Sensory and motivational processes (pp. 97-135). Hillsdale, NJ: Erlbaum.

Lang, P. J., Greenwald, M. K., Bradley, M. M., \& Hamm, A. O. (1993). Looking at pictures: Affective, facial, visceral, and behavioral reactions. Psychophysiology, 30, 261-273.

Laux, L., Glanzmann, P. S. P., \& Spielberger, C. D. (1981). Das StateTrait-Angstinventar (STAI). Weinheim: Beltz.

Linton, M. (1982). Transformations of memory in everyday life. In U. Neisser (Ed.), Memory observed: Remembering in natural contexts (pp. 77-91). San Francisco, CA: Freeman.

MacLeod, C., Mathews, A., \& Tata, P. (1986). Attentional bias in emotional disorders. Journal of Abnormal Psychology, 95, 15-20.

Martin, M., Williams, R. M., \& Clark, D. M. (1991). Does anxiety lead to selective processing of threat-related information? Behaviour Research and Therapy, 29, 147-160.

McKenna, F. P., \& Sharma, D. (1995). Intrusive cognitions: An investigation of the emotional Stroop task. Journal of Experimental Psychology: Learning, Memory \& Cognition, 21, 1595-1607.

McKenna, F. P., \& Sharma, D. (2004). Reversing the emotional Stroop effect: The role of fast and slow components. Journal of Experimental Psychology: Learning Memory and Cognition, 30, 382392.

Naccache, L., Gaillard, R., Adam, C., Hasboun, D., Clémenceau, S., Baulac, M., et al. (2005). A direct intracrancial record of emotions evoked by subliminal words. Proceedings of the National Academy of Sciences of the United States of America, 102, 77137717.

Pratto, F., \& John, O. P. (1991). Automatic vigilance: The attentiongrabbing power of negative social information. Journal of Personality and Social Psychology, 61, 380-391. 
Pratto, F. (1994). Consciousness and automatic evaluation. In P. M. Niedenthal \& S. Kitayama (Eds.), The heart's eye: Emotional influences in perception and attention (pp. 115-143). San Diego, CA: Academic Press.

Richards, A., French, C. C., Johnson, W., Naparstek, J., \& Williams, J. (1992). Effects of mood manipulation and anxiety on performance of an emotional Stroop task. British Journal of Psychology, 83, 479-491.

Rubin, D. C. (2005). Autobiographical memory tasks in cognitive research. In A. Wenzel \& D. C. Rubin (Eds.), Cognitive methods and their application to clinical research (pp. 219-242). Washington, DC: American Psychological Association.

Ruiz-Caballero, J. A., \& Bernandez, J. (1997). Anxiety and attention. Is there an attentional bias for positive words. The Journal of General Psychology, 124, 194-211.

Robinson, M. D., \& Compton, R. J. (2006). The automaticity of affective reactions: Stimulus valence, arousal, and lateral spatial attention. Social Cognition, 24, 469-495.
Rusting, C. L. (1998). Personality, mood, and cognitive processing of emotional information: three conceptual frameworks. Psychological Bulletin, 124, 165-196.

Schimmack, U. (2005). Attentional interference effects of emotional pictures: Threat, negativity, or arousal? Emotion, 5, 55-66.

Sharma, D., \& McKenna, F. P. (2001). The role of time pressure on the emotional Stroop task. British Journal of Psychology, 92, 471481.

Waagenar, W. A. (1986). My memory: A study of autobiographical over six years. Cognitive Psychology, 18, 225-252.

Williams, J. M. G., Mathews, A., \& MacLeod, C. (1996). The emotional Stroop task and psychopathology. Psychological Bulletin, $120,3-24$

Williams, J. M. G., Watts, F. N., MacLeod, C., \& Mathews, A. (1997). Cognitive psychology and emotional disorders (2nd ed.). New York: Wiley. 